\title{
Taste Disorders Are Partly Genetically Determined: Role of the TAS2R38 Gene, a Pilot Study
}

\author{
Melania Melis, PhD; Lisa Grzeschuchna, MS; Giorgia Sollai, PhD; Thomas Hummel, MD (D; \\ Iole Tomassini Barbarossa, $\mathrm{PhD}$ (1)
}

\begin{abstract}
Objectives/Hypothesis: Taste sensitivity varies greatly among individuals influencing eating behavior and health, consequently the disorders of this sense can affect the quality of life. The ability to perceive the bitter of thiourea compounds, such as phenylthiocarbamide (PTC), has been largely reported as a marker of the general taste sensitivity, food preferences, and health. PTC sensitivity is mediated by the TAS2R38 receptor and its genetic common variants. We study the role of the TAS2R38 receptor in taste disorders with the aim of understanding if these can be genetically determined.

Study Design: Prospective cohort study.

Methods: Differences in the PTC responsiveness between the patients cohort and healthy controls were assessed. All subjects received standardized tests for smell and taste function and were genotyped for the TAS2R38 gene.

Results: PAV/PAV homozygous patients gave high PTC ratings, whereas PAV/AVI genotypes reported lower values, which are similar to those determined in AVI/AVI or rare genotypes. In addition, the patients cohort did not meet the Hardy-Weinberg equilibrium at the TAS2R38 locus, showing a very low frequency of subjects carrying the PAV/AVI diplotype. Independently, in healthy controls who were in equilibrium at the locus, PAV/PAV homozygous and heterozygous rated PTC bitterness higher compared to AVI/AVI or rare genotypes.

Conclusions: Our findings, by showing that an only taster haplotype (PAV) is not sufficient to evoke high responses of TAS2R38 receptor in patients with taste disorders, suggest that the genetic constitution may represent a risk factor for the development of taste disorders.
\end{abstract}

Key Words: Phenylthiocarbamide responsiveness, TAS2R38 bitter receptor, taste disorders.

Level of Evidence: $2 c$

Laryngoscope, 00:1-6, 2019

\section{INTRODUCTION}

In humans, taste and smell play an important role in the interaction of individuals with the environment, affecting behavior and memory processes. ${ }^{1}$ These senses

Additional supporting information may be found online in the Supporting Information section at the end of the article.

From the Department of Biomedical Sciences, University of Cagliari (M.M., G.S., I.T.B.), Monserrato, Italy; and the Department of Otorhinolaryngology, Smell and Taste Clinic, Dresden University of Technology (L.G., T.H.), Dresden, Germany.

Editor's Note: This Manuscript was accepted for publication on December 4, 2018

M.M. and L.G. contributed equally to this work. T.H. and I.T.B. are cosenior authors.

All authors have made substantial contributions to this project including contributions to conception and design, or data acquisition, or data analysis and interpretation. All authors were involved in drafting the manuscript or revising it critically for important intellectual content. All authors have given final approval of the manuscript as submitted for publication and take responsibility for content. The authors agree to be accountable for all aspects of the work in ensuring that questions related to the accuracy or integrity of any part of the work are appropriately investigated and resolved.

This study was supported by a grant from the University of Cagliari (Fondo Integrativo per la Ricerca [FIR 2016-2017]).

The authors have no other funding, financial relationships, or conflicts of interest to disclose.

Send correspondence to Iole Tomassini Barbarossa, Department of Biomedical Sciences, Section of Physiology, S.P. 8, 09042 Monserrato, CA, Italy. E-mail: tomassin@unica.it; and Thomas Hummel, Department of Otorhinolaryngology, Smell and Taste Clinic, TU Dresden, Fetscherstrasse 74, 01307 Dresden, Germany. E-mail: thummel@mail.zih.tu-dresden.de

DOI: 10.1002/lary.27828 guide organisms to identify and consume nutrients while avoiding toxins and indigestible materials. ${ }^{2-4}$ Hence, it is not surprising that olfactory and taste disorders can have important effects on the quality of life, the importance of which we can realize only when they are lost. ${ }^{5,6}$ Moreover, unexplained smell impairments are acknowledged to be very early biomarkers of the neurodegenerative diseases or mortality, ${ }^{7}$ and recently it has been reported that also taste disruption may represent a risk factor associated for these disorders. ${ }^{8}$

Taste is the sensory modality that acts as the ultimate checkpoint for food acceptance or rejection. ${ }^{2}$ It is generally assumed that the taste system can differentiate the five classic taste qualities (sweet, umami, sour, salty, and bitter), which represent different physiological requirements or pose potential eating hazards, and other additional qualities such as fatty and metallic. Taste sensitivity varies greatly among individuals influencing food choice and health. ${ }^{9}$ This individual variability depends on many factors including the properties of saliva ${ }^{10}$ morphology of taste papillae,${ }^{11}$ gender and age,${ }^{12}$ cultural and social reasons, but there is also a significant genetic component, which could be the result of evolutionary adaptation mechanisms. ${ }^{13}$ The genetic ability to perceive the bitter taste of thiourea compounds, such as phenylthiocarbamide (PTC) and 6-n-propylthiouracil (PROP), has been broadly reported as an oral marker of food preferences and physiological mechanisms. ${ }^{9,14,15}$ This report is based on results that have 
shown relationships between taste sensitivity to PTC/PROP and to other taste stimuli, ${ }^{16-24}$ food-liking patterns, ${ }^{24-28}$ or health markers such as body mass index, ${ }^{27,29}$ antioxidant status, ${ }^{30}$ colonic neoplasm risk, ${ }^{31}$ smoking behavior, ${ }^{32}$ and predisposition to respiratory infections. ${ }^{33}$

PTC and PROP taste perception are mediated by TAS2R38 receptor, which is expressed by the TAS2R38 gene residing on chromosome $7 .^{34}$ The allelic diversity of this gene can explain most of individual differences in PTC/PROP tasting. ${ }^{34,35}$ The three single nucleotide polymorphism (SNPs) of TAS2R38 (rs713598, rs1726866, and rs10246939) give rise at three amino acid substitutions (proline to alanine at residue 49, alanine to valine at residue 262, and valine to isoleucine at residue 296), which result in two major haplotypes (the taster dominant variant, PAV, and the nontaster recessive variant, AVI $)^{18,35}$ and rare haplotypes (AAI, AAV, and PVI), which contribute to intermediate sensitivity. ${ }^{36,37}$ Bitter taste receptors can detect bitter compounds, not only in the oral cavity, but also in many extraoral tissues. ${ }^{38}$ However, the functions of these extraoral receptors are only partially known. Nevertheless, it is known that TAS2R38 plays an important role in the innate defense in the human respiratory tract because it can be activated by the bitter quorum-sensing molecules secreted by bacteria, and its allelic diversity contributes to different susceptibility that people have to infections of the respiratory system. ${ }^{33,39}$ Although the role of the bitter taste receptor TAS2R38 in chronic rhinosinusitis has already been shown by preliminary clinical data, ${ }^{39}$ further prospective clinical studies on the influence of the TAS2R38 genotype and chronic rhinosinusitis susceptibility are needed, also in relation with taste impairments.

From this background, the purpose of this work was to study the role of the TAS2R38 receptor and its variants with a different affinity for the stimulus in a cohort of patients with taste disorders for the four basic tastes. ${ }^{40}$ To this aim, we assessed the differences, between the patients cohort and healthy controls, in taste ratings for the specific stimulus, PTC, and in genotype distribution, haplotype frequencies, and Hardy-Weinberg equilibrium at the TAS2R38 locus.

\section{MATERIALS AND METHODS}

\section{Subjects}

Seventy-nine Caucasian subjects ( 28 males and 51 females) were recruited at the Smell and Taste Clinic at the Department of Otorhinolaryngology of the Dresden University of Technology, Dresden, Germany. They consisted of two groups: patients ( $\mathrm{n}=33$; six male, 27 female; age $63.87 \pm 2.71$ years) and healthy controls ( $\mathrm{n}=46 ; 22$ male, 24 female; age $47.12 \pm 2.45$ years). The exclusion criteria involved major diseases (e.g., diabetes and kidney disease), pregnancy or lactation, or food allergies. All patients had taste problems, meaning they had either quantitative taste loss or qualitative taste problems (i.e., taste distortions). They were included in the study based on their complaints. It is important to note that patients with taste disorders were not devoid of taste function, and most of them were able to perceive the four basic tastes.

All participants received a detailed otorhinolaryngological examination including nasal endoscopy, a structured history was taken, and all participants received standardized tests for smell and taste function. ${ }^{41}$ Healthy controls did not exhibit major otolaryngologic disorders; they did not mention any disturbances of smell or taste.

All subjects were informed (verbally and in writing) regarding the aim and procedure of the study and signed a consent form. The present study was conducted in accordance with the guidelines of the Declaration of Helsinki of 1975 (revised in 1983), and all procedures were approved by the ethics committee at the University Hospital of the Dresden University of Technology (protocol number EK286112008).

\section{Experimental Protocol \\ Taste measurements}

TASTE SPRAYS. Gustatory function was screened with suprathreshold tastants sprayed onto the tongue, so-called "taste sprays," which had to be identified as sweet, sour, salty, or bitter. $^{42}$ This test provided information whether the patient was able to recognize and differentiate different taste qualities.

TASTE STRIPS. Taste function was furthermore assessed using the validated "taste strips," which are spoonshaped filter papers impregnated with the four basic taste qualities (sweet, sour, salty, bitter) in the following concentrations: sweet: $0.4,0.2,0.1,0.05 \mathrm{~g} / \mathrm{mL}$ sucrose; sour: $0.3,0.165,0.09$, $0.05 \mathrm{~g} / \mathrm{mL}$ citric acid; salty: $0.25,0.1,0.04,0.016 \mathrm{~g} / \mathrm{mL}$ sodium chloride; bitter: $0.006,0.0024,0.0009,0.0004 \mathrm{~g} / \mathrm{mL}$ quinine hydrochloride. The taste strips were placed on both sides of the anterior third of the extended tongue (mouth opened) in increasing concentrations. Taste quality had to be identified from a list of four possible answers (sweet, sour, salty, and bitter) in a forced choice procedure. Taste qualities were presented in a random fashion including also the side of presentation (left/right). Before every new testing, participants were asked to rinse their mouth with tap water. The number of correctly identified tastes was summed up to a score for each taste quality as well as a general taste score. ${ }^{43}$

OLFACTORY FUNCTION. In addition, orthonasal olfactory function was measured by means of the extended Sniffin' Sticks test, ${ }^{44}$ which is based on odor-containing felt-tip pens. This test consists of three subtests: threshold, discrimination, and identification (TDI) test. For each subtest, the pen's cap was removed, and its felt tip was presented about $2 \mathrm{~cm}$ in front of both nostrils of the subject for about 3 seconds. The testing procedure began with the threshold part in a triple-forced choice paradigm, where participants had to discriminate the odor (phenyl ethyl alcohol [PEA]) from two blanks (filled with solvent propylene glycol). Starting with the lowest PEA concentration, a staircase paradigm was used where two correct or one incorrect answer led to a decrease or increase of concentration, the socalled turning point. The resulting threshold score was the mean of the last four turning points in the staircase. The next subtest performed was the discrimination test, where two pens had the same odor, whereas the other one had a different scent that had to be identified. The last task was the identification test, where the subject was asked to choose the object that describes the odor the best using a four-alternative forced choice from flash cards that had both the picture and name of the object. The scores of the olfactory subtests were then summed up building the overall TDI score.

PTC tasting. Bitter-taster status was determined using a suprathreshold method. Filter paper impregnated with PTC was placed in the mouth (at the anterior tongue region) of participants (phenylthiocarbamide test paper; Sensonics, Philadelphia, PA), 
who were then asked to rate the intensity of the bitter taste on a visual analog scale from 0 to $10(0=$ no taste at all, $10=$ extremely strong taste).

\section{Saliva Collection}

Saliva collection was performed before the other tests so that the following olfactory and gustatory stimulation could not affect salivation. Participants had been asked not to drink, eat, or smoke 1 hour before the examination. Unstimulated saliva was collected for 5 minutes with participants spitting into plastic containers. To determine salivary flow rates, the amount of collected saliva was weighed. The secretion rate was calculated, and the plastic containers were frozen to preserve the material until molecular analysis.

\section{Molecular Analysis}

DNA was extracted from saliva samples using the QIAamp DNA Mini Kit (QIAGEN Srl, Milan, Italy) according to the manufacturer's instructions. This kit is specific to extract DNA from saliva samples. The concentration of purified DNA was estimated by measuring the optical density at $260 \mathrm{~nm}$ with an Agilent Cary 60 UV-Vis Spectrophotometer (Agilent Technologies Australia Pty. Ltd., Mulgrave, Victoria, Australia). Subjects were genotyped for three SNPs (rs713598, rs1726866, and $r s 10246939)$ of TAS2R38, at base pairs (bp) $145(\mathrm{C} / \mathrm{G}), 785(\mathrm{C} / \mathrm{T})$, and $886(\mathrm{G} / \mathrm{A})$, respectively. The three SNPs of TAS2R38 consist of three amino acid substitutions (Pro49Ala, Ala262Val, and Val296Ile), which give rise to two major haplotypes, PAV (the dominant taster variant) and AVI (the nontaster recessive one) and three rare haplotypes (AAI, AAV, and PVI). A polymerase chain reaction was employed to amplify the short region including the first polymorphism ( $r$ 7 713598) at nt 145: a 221-bp fragment was amplified with forward 5'-CCTTCGTTTTCTTGGTGAATTT TTGGGATGTAGTG AAGAGGCGG-3' and reverse 5'-AGGTTGGCTTGGTTTGCAATC ATC-3' primers. Amplified samples were digested with HaeIII, according to our previous work. ${ }^{11}$ The HaeIII digest the C SNP. For the rs1726866 and rs10246939 SNPs, TaqMan SNP Genotyping Assay (C_9506827_10 for the rs1726866 assay and C_95068 26_10 for the rs10246939 assay; Applied Biosystems by Life-Technologies Italia, Europe BV, Monza, Italy $)^{45-47}$ was used according to the manufacturer's specifications. Replicates and positive and negative controls were included in all reactions.

\section{Statistical Analyses}

Differences between patients and healthy controls on genotype distribution and haplotype frequencies at the TAS $2 R 38$ locus were compared using the Fisher method (Genepop software version 4.2; http://genepop.curtin.edu.au/genepop_op3.html). ${ }^{48}$ Hardy-Weinberg equilibrium at the TAS2R38 was calculated for patients and healthy controls by using the Hardy-Weinberg exact test (Genepop program version 4.2; http://genepop.curtin.edu. au/genepop_op1.html), to verify if each population is behaving like a single randomly mating unit without intense viability selection acting on the sampled locus. ${ }^{49}$ The Hardy-Weinberg expected frequency for each genotype of TAS2R38 was also calculated for $\mathrm{p} 2,2 \mathrm{pq}$, and $\mathrm{q} 2$ in the two populations by using the Levene correction (Genepop program version 4.2; (http://genepop. curtin.edu.au/genepop_op5.html). This equation permits relating allele frequencies to genotype frequencies for each population.

One-way analysis of variance (ANOVA) was used to compare the ratings of the PTC perceived intensity according to TAS2R38 genotypes in patients and healthy controls. Subjects with rare haplotypes was included in a single group because there were too few subjects for each rare genotype. PTC rating differences among TAS2R38 groups, in patients and healthy controls, were also evaluated by two-way analysis of covariance (ANCOVA), in which age and gender were considered as covariates. Post hoc comparisons were conducted with the Fisher least significant difference (LSD) test, unless the assumption of homogeneity of variance was violated, in which case the Duncan test was used. Statistical analyses were conducted using STATISTICA for Windows (version 7; StatSoft Inc., Tulsa, OK). $P$ values $\leq .05$ were considered significant.

\section{RESULTS}

Table I shows the mean values \pm standard error of taste and smell function evaluations determined in patients and healthy controls. On a clearly suprathreshold level, patients and controls were able to identify sweet, sour, salty, and bitter with no significant difference between groups $(P<.05)$. However, one-way ANOVA showed the more detailed analyses with taste strips and Sniffin' Sticks (TDI) that healthy controls scored higher than patients (ANOVA $F[1,71] \geq 12.70 ; P \leq .00066$ ).

Molecular analysis at the three SNPs of the TAS2R38 locus identified, in the group of healthy controls, eight subjects who were PAV homozygous, 18 were heterozygous, and 13 were AVI homozygous, whereas in the group of patients, eight subjects were PAV homozygous, seven were heterozygous, and 10 were AVI homozygous. Rare haplotypes were found in eight subjects of the patient group (six carried the AAV/AVI genotype, one the PAV/AAV genotype, and one the PVI/AVI genotype), and in seven healthy controls (three had AAV/AVI genotype, two the PAV/AAV genotype, one the PAV/PVI genotype, and one the AAI/AVI genotype) (Table II). Although the heterozygous subjects were uncommon in the patient group, only $21 \%$ of them carried this genotype. Dominant and recessive homozygous subjects were more, compared to healthy controls, and no significant differences were found between patients and healthy controls based on the genotype distribution and allele frequency of the TAS2R38 locus $\left(\chi^{2}=0.274 ; P=.871\right.$ and $\chi^{2}=0.308 ; P$ $=.857 ;$ Fisher method). The statistical differences with and without inclusion of rare haplotypes in the analysis were the same. In addition, the Hardy-Weinberg exact test showed that the patients cohort did not meet the Hardy-Weinberg equilibrium for TAS2R38 $(P=.023)$, whereas the healthy controls sample met the HardyWeinberg equilibrium $(P=.938)$ (Table III). In addition,

TABLE I.

Taste and Smell Function Assessments.

\begin{tabular}{lcc}
\hline & Patients & Healthy Controls \\
\hline Taste sprays & $3.57 \pm 0.13$ & $3.81 \pm 0.12$ \\
Taste strips & $9.66 \pm 0.45^{*}$ & $11.84 \pm 0.41^{*}$ \\
Sniffin' Sticks (TDI score) & $28.01 \pm 1.19^{*}$ & $33.80 \pm 1.10^{*}$ \\
\hline \hline
\end{tabular}

All values are mean \pm standard error.

*Differences between the patients cohort and healthy controls evaluated by one-way analysis of variance $F(1,71) \geq 12.70 ; P \leq .00066$ (patients, $n=33$; healthy controls, $n=46$ ).

TDI = threshold, discrimination, and identification. 
TABLE II.

Genotype Distribution and Haplotype Frequencies of Polymorphisms of TAS2R38 in the Patients and Healthy Controls.

\begin{tabular}{lcccccc}
\hline & \multicolumn{2}{c}{ Patients } & & \multicolumn{2}{c}{ Healthy Controls } & \\
\cline { 2 - 3 } TAS2R38 & $n$ & $\%$ & & $n$ & & P Value* \\
Genotype & & & & & & \\
PAV/PAV & 8 & 24.24 & & 8 & 17.39 & .871 \\
PAV/AVI & 7 & 21.21 & & 18 & 39.13 & \\
AVI/AVI & 10 & 30.30 & & 13 & 28.26 & \\
AAV/AVI & 6 & 10.09 & & 3 & 6.52 & \\
PAV/AAV & 1 & 3.03 & & 2 & 4,35 & \\
PAV/PVI & 0 & 0 & & 1 & 2.17 & \\
AAI/AVI & 0 & 0 & & 1 & 2.17 & \\
PVI/AVI & 1 & 3.03 & & 0 & 0 & \\
Haplotype & & & & & \\
PAV & 24 & 36.36 & & 37 & 40.22 & \\
AVI & 34 & 51.51 & & 48 & 52.17 & \\
AAV & 7 & 10.60 & & 5 & 5.43 & \\
PVI & 1 & 1.51 & & 1 & 1.08 & \\
AAI & 0 & 0 & & 1 & 1.08 &
\end{tabular}

$* P$ values were derived from the Fisher method (patients, $\mathrm{n}=33$; healthy controls, $n=46$ ).

the Levene correction showed that, in the patients cohort, the expected frequency for PAV/PAV, AVI/AVI, and rare genotype was lower than that observed. In contrast, the expected frequency for heterozygous genotype was higher than that observed one. Specifically, for the PAV/PAV genotype the expected frequency was 4.25 and the observed one was 8 , for the heterozygous genotype the expected frequency was 12.55 and the observed one was 7, for the AVI/AVI genotype the expected frequency was 7.57 and the observed one was 10 , and for subjects with the rare haplotype the expected frequency was 5.57 and the observed one was 8 . On the other hand, in healthy controls the expected and observed frequencies for different TAS2R38 genotypes were overlapping.

One-way ANOVA showed that PTC rating varied with TAS2R38 genotypes $(F[3,42]=3.31 ; P=.029)$ in

TABLE III.

Hardy-Weinberg Equilibrium Results at the TAS2R38 for Patients and Healthy Controls.

\begin{tabular}{|c|c|c|c|c|c|c|}
\hline \multirow[b]{2}{*}{ TAS2R38 } & \multicolumn{3}{|l|}{ Patients } & \multicolumn{3}{|c|}{ Healthy Controls } \\
\hline & Observed & Expected & $P$ Value* & Observed & Expected & $P$ Value* \\
\hline Genotype & & & .023 & & & .938 \\
\hline PAV/PAV & 8 & 4.25 & & 8 & 7.32 & \\
\hline PAV/AVI & 7 & 12.55 & & 18 & 19.52 & \\
\hline $\mathrm{AVI} / \mathrm{AVI}$ & 10 & 7.57 & & 13 & 12.40 & \\
\hline Rare & 8 & 5.57 & & 7 & 6.76 & \\
\hline
\end{tabular}

$* P$ values relative to Hardy-Weinberg equilibrium at the TAS2R38 for the patients cohort and healthy controls were derived from the HardyWeinberg exact test (patients, $n=33$; healthy controls, $n=46$ ). The expected frequency for each genotype of TAS2R38 was calculated by using the Levene correction.

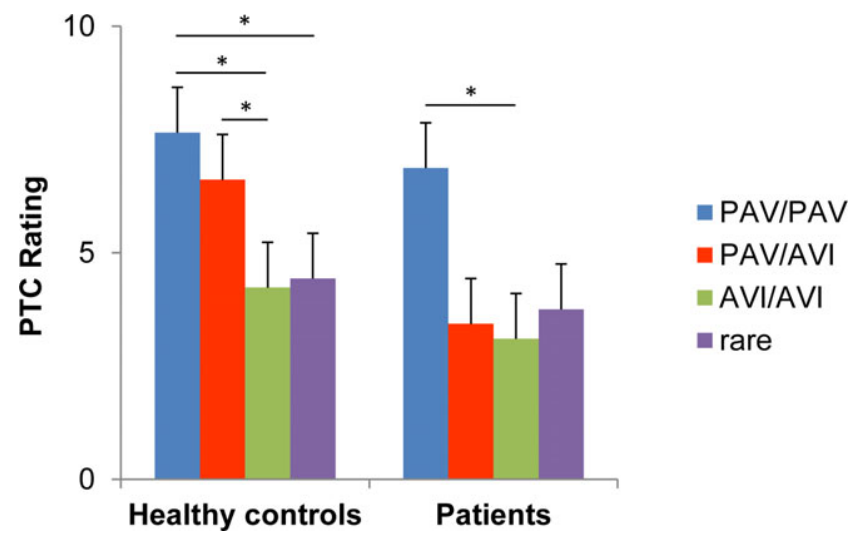

Fig. 1. Phenylthiocarbamide (PTC) ratings of subjects genotyped for TAS2R38 of the two groups: patients cohort $(n=33)$ and healthy controls $(n=46)$. All values are mean \pm standard error. One-way analysis of variance was used to compare values across genotype groups (patients: $F[3,29]=2.1282 ; P=.1183$; healthy controls: $F[3,42]=3.31 ; P=.029)$. *Significant difference $(P \leq .036$; Fisher least significant difference test). [Color figure can be viewed in the online issue, which is available at www.laryngoscope.com.]

healthy controls, but not in patients $(F[3,29]=2.1282$; $P=.1183$ ) (Fig. 1). Post hoc comparisons showed that mean ratings were statistically higher in the healthy controls who have the PAV/PAV and PAV/AVI genotype with respect to those who have the AVI/AVI genotype $(P \leq .033$; Fisher LSD). PAV/PAV healthy controls showed higher ratings also than those with rare haplotype $(P=.036$; Fisher LSD). Independently, in the patients cohort, only subjects with the PAV/PAV genotype show higher ratings with respect to the AVI/AVI genotypes $(P \leq .027$; Fisher LSD), whereas the PAV/AVI patients show similar ratings to those of patients with the AVI/AVI or rare genotype $(P>.05)$. A two-way ANCOVA confirmed these results after controlling for age and gender (ratings differences related to TAS2R38 in healthy controls: PAV/PAV genotypes and PAV/AVI genotypes > AVI/AVI genotypes and PAV/PAV genotypes $>$ rare haplotype, $P \leq .0441$, Fisher LSD; ratings differences related to TAS2R38 in the patients cohort: PAV/PAV genotypes > PAV/AVI genotypes and AVI/AVI genotypes, $P<.037$, Fisher LSD). Post hoc comparison also showed that PAV/AVI healthy controls had PTC ratings higher than those of patients with the same genotype $(P=.026$; Fisher LSD). PTC ratings of patients and heathy controls according TAS2R38, including each rare haplotype, are shown in Supporting Figure 1 in the online version of this article.

\section{DISCUSSION}

Previous research has shown a large genotypic overlap between the PTC/PROP medium and supertaster groups, ${ }^{18,35}$ with significant numbers of supertaster subjects carrying the genotype heterozygous PAV/AVI. Another study suggested that the presence of two PAV haplotypes (as opposed to one) confers no additional benefit for perceiving more PROP bitterness intensity. ${ }^{46}$ In the present work, we studied the role of the TAS2R38 receptor and its variants with different affinity for the 
stimulus in a cohort of patients who were diagnosed for taste disorders for the four basic tastes compared to healthy controls. Our results showed that only PAV/PAV homozygous patients gave high PTC ratings, whereas PAV/AVI genotypes reported lower values, which are similar to those determined in patients with the AVI/AVI or rare genotype.

Lee et al. ${ }^{33}$ reported that PAV/PAV super-tasters are less susceptible to sinonasal infections than PAV/AVI or AVI/AVI patients who have impaired TAS2R38-dependent responses. In agreement with this study, our results, indicate that an only taster haplotype (PAV) is not sufficient to evoke high responses of the TAS2R38 receptor in the patients with taste disorders. This could be due to their bitter perception, which is compromised, and thus, they have trouble perceiving PTC. In contrast, patients with the $\mathrm{PAV} / \mathrm{PAV}$ genotype rated the tastes as more intense, indicating that they were not so severely affected by their taste disorder, probably because their basic sensitivity is much higher. These data may also suggest that the PAV/AVI and AVI/AVI patients do not recover completely following treatments or not as fast as the PAV/PAV patients, possibly suggesting that a different prognosis should be assigned to these patients based on the TAS2R38 genotype of the patient.

On the other hand, in the cohort used as the control in the present study, we found that subjects with PAV/ PAV homozygous and heterozygous genotypes rated the intensity of PTC bitterness higher compared to AVI/AVI or rare genotypes. These results are consistent with what is reported in studies on healthy subjects, ${ }^{46}$ and thus validates the use of this cohort as a control.

Our results showed no significant differences based on genotype distribution and haplotype frequencies between the two populations compared, suggesting that patient and control samples could be considered as a unique group. However, we found that the cohort of healthy subjects did no show deviation from HardyWeinberg equilibrium, confirming no population subdivision, viability selection, or systematic mating. On the contrary, the deviation from equilibrium determined in the patients cohort could suggest a viability selection at the TAS2R38 locus. The frequency of subjects carrying PAV/AVI diplotype in the patients cohort was much lower (only 21\%) than what has been determined in other Caucasian populations. ${ }^{50}$ This indicates that this specific genotype may constitute a protective factor to develop dysgeusia. Nevertheless, if we had used a patients population with complete taste loss we probably would have found differences in genotype distribution and haplotype frequencies, with respect to healthy controls.

\section{CONCLUSION}

These findings, which should be confirmed in a larger population, represent an important first step in elucidating the role of TAS2R38 in taste disorders. These results, by showing that an only haplotype (PAV) in TAS2R38 is not sufficient to exhibit high taste responses, suggest that the nontasting variant (AVI) in this locus might represent a risk factor to develop taste disorders.
Also, based on the results that have suggested the TAS2R38 pathway as a potential therapeutic target, ${ }^{39}$ our studies are in progress to investigate the hypothesis that PAV/AVI and AVI/AVI patients, who should be not very responsive, may have a different prognosis than PAV/PAV patients.

\section{BIBLIOGRAPHY}

1. Huart C, Collet $\mathrm{S}$, Rombaux P. Chemosensory pathways: from periphery to cortex. B-ENT 2009;5(suppl 13):3-9.

2. Scott K. Taste recognition: food for thought. Neuron $2005 ; 48: 455-464$.

3. Chaudhari N, Roper SD. The cell biology of taste. J Cell Biol 2010;190: $285-296$.

4. Stevenson RJ. An initial evaluation of the functions of human olfaction. Chem Senses 2010;35:3-20.

5. Hummel T, Landis BN, Hüttenbrink K-B. Smell and taste disorders. GMS Curr Top Otorhinolaryngol Head Neck Surg 2011;10:Doc04.

6. Croy I, Nordin S, Hummel T. Olfactory disorders and quality of life-an updated review. Chem Senses 2014;39:185-194.

7. Pinto JM, Wroblewski KE, Kern DW, Schumm LP, McClintock MK. Olfactory dysfunction predicts 5-year mortality in older adults. PLoS One 2014; 9:e107541.

8. Cossu G, Melis M, Sarchioto M, et al. 6-n-propylthiouracil taste disruption and TAS2R38 nontasting form in Parkinson's disease. Mov Disord 2018; 33:1331-1339.

9. Tepper BJ. Nutritional implications of genetic taste variation: the role of PROP sensitivity and other taste phenotypes. Annu Rev Nutr 2008;28: 367-388.

10. Melis M, Arca M, Aragoni MC, et al. Dose-dependent effects of L-arginine on PROP bitterness intensity and latency and characteristics of the chemical interaction between PROP and L-arginine. PLoS One 2015;10: e0131104.

11. Melis M, Atzori E, Cabras S, et al. The gustin (CA6) gene polymorphism, rs2274333 (A/G), as a mechanistic link between PROP tasting and fungiform taste papilla density and maintenance. PLoS One 2013;8:e74151.

12. Tepper BJ, Melis M, Koelliker Y, Gasparini P, Ahijevych KL, Tomassini Barbarossa I. Factors influencing the phenotypic characterization of the oral marker, PROP. Nutrients 2017;9(12).

13. Soranzo N, Bufe B, Sabeti PC, et al. Positive selection on a high-sensitivity allele of the human bitter-taste receptor TAS2R16. Curr Biol 2005;15: 1257-1265.

14. Tepper BJ, Banni S, Melis M, Crnjar R, Tomassini Barbarossa I. Genetic sensitivity to the bitter taste of 6-n-propylthiouracil (PROP) and its association with physiological mechanisms controlling body mass index (BMI). Nutrients 2014;6:3363-3381.

15. Doty RL, De Fonte TP. Relationship of phenylthiocarbamide (PTC) taster status to olfactory and gustatory function in patients with chemosensory disturbances. Chem Senses 2016;41:685-696.

16. Yeomans MR, Tepper BJ, Rietzschel J, Prescott J. Human hedonic responses to sweetness: role of taste genetics and anatomy. Physiol Behav 2007;91:264-273.

17. Prescott J, Soo J, Campbell H, Roberts C. Responses of PROP taster groups to variations in sensory qualities within foods and beverages. Physiol Behav 2004;82:459-469.

18. Duffy VB, Davidson AC, Kidd JR, et al. Bitter receptor gene (TAS2R38), 6-n-propylthiouracil (PROP) bitterness and alcohol intake. Alcohol Clin Exp Res 2004;28:1629-1637.

19. Melis M, Yousaf NY, Mattes MZ, et al. Sensory perception of and salivary protein response to astringency as a function of the 6-n-propylthioural (PROP) bitter-taste phenotype. Physiol Behav 2017;173:163-173.

20. Melis M, Tomassini Barbarossa I. Taste perception of sweet, sour, salty, bitter, and umami and changes due to L-arginine supplementation, as a function of genetic ability to taste 6-n-propylthiouracil. Nutrients 2017; $9(6)$.

21. Bartoshuk LM. The biological basis of food perception and acceptance. Food Qual Prefer 1993;4:21-32.

22. Bartoshuk LM, Duffy VB, Lucchina LA, Prutkin J, Fast K. PROP (6-n-propylthiouracil) supertasters and the saltiness of $\mathrm{NaCl}$. Ann N Y Acad Sci 1998;855:793-796.

23. Melis M, Sollai G, Muroni P, Crnjar R, Barbarossa IT. Associations between orosensory perception of oleic acid, the common single nucleotide polymorphisms (rs1761667 and rs1527483) in the CD36 gene, and 6-npropylthiouracil (PROP) tasting. Nutrients 2015;7:2068-2084.

24. Hayes JE, Duffy VB. Revisiting sugar-fat mixtures: sweetness and creaminess vary with phenotypic markers of oral sensation. Chem Senses 2007; 32:225-236.

25. Keller KL, Steinmann L, Nurse RJ, Tepper BJ. Genetic taste sensitivity to 6-n-propylthiouracil influences food preference and reported intake in preschool children. Appetite 2002;38:3-12.

26. Dinehart ME, Hayes JE, Bartoshuk LM, Lanier SL, Duffy VB. Bitter taste markers explain variability in vegetable sweetness, bitterness, and intake. Physiol Behav 2006;87:304-313. 
27. Tepper BJ, Neilland M, Ullrich NV, Koelliker Y, Belzer LM. Greater energy intake from a buffet meal in lean, young women is associated with the 6-npropylthiouracil (PROP) non-taster phenotype. Appetite 2011;56:104-110.

28. Robino A, Mezzavilla M, Pirastu N, Dognini M, Tepper BJ, Gasparini P. A population-based approach to study the impact of PROP perception on food liking in populations along the Silk Road. PLoS One 2014;9:e91716.

29. Tepper BJ, Koelliker Y, Zhao L, et al. Variation in the bitter-taste receptor gene TAS2R38, and adiposity in a genetically isolated population in Southern Italy. Obesity 2008;16:2289-2295.

30. Tepper BJ, Williams TZ, Burgess JR, Antalis CJ, Mattes RD. Genetic variation in bitter taste and plasma markers of anti-oxidant status in college women. Int J Food Sci Nutr 2009;60(suppl 2):35-45.

31. Lucock M, Ng X, Boyd L, et al. TAS2R38 bitter taste genetics, dietary vitamin $\mathrm{C}$, and both natural and synthetic dietary folic acid predict folate status, a key micronutrient in the pathoaetiology of adenomatous polyps. Food Funct 2011;2:457-465.

32. Risso DS, Kozlitina J, Sainz E, et al. Genetic variation in the TAS2R38 bitter taste receptor and smoking behaviors. PLoS One 2016;11:e0164157.

33. Lee RJ, Xiong G, Kofonow JM, et al. T2R38 taste receptor polymorphisms underlie susceptibility to upper respiratory infection. J Clin Invest 2012; 122:4145-4159.

34. Kim UK, Jorgenson E, Coon H, Leppert M, Risch N, Drayna D. Positional cloning of the human quantitative trait locus underlying taste sensitivity to phenylthiocarbamide. Science 2003;299:1221-1225.

35. Bufe B, Breslin PA, Kuhn C, et al. The molecular basis of individual differences in phenylthiocarbamide and propylthiouracil bitterness perception. Curr Biol 2005;15:322-327.

36. Wooding S, Kim UK, Bamshad MJ, Larsen J, Jorde LB, Drayna D. Natura selection and molecular evolution in PTC, a bitter-taste receptor gene. Am J Hum Genet 2004;74:637-646.

37. Boxer EE, Garneau NL. Rare haplotypes of the gene TAS2R38 confer bitter taste sensitivity in humans. SpringerPlus 2015;4:505.

38. Yamamoto K, Ishimaru Y. Oral and extra-oral taste perception. Semin Cell Dev Biol 2013;24:240-246.
39. Lee RJ, Cohen NA. Role of the bitter taste receptor T2R38 in upper respiratory infection and chronic rhinosinusitis. Curr Opin Allergy Clin Immunol $2015 ; 15: 14-20$

40. Fark T, Hummel C, Hahner A, Nin T, Hummel T. Characteristics of taste disorders. Eur Arch Otorhinolaryngol 2013;270:1855-1860.

41. Welge-Luessen A, Leopold DA, Miwa T. Smell and taste disordersdiagnostic and clinical work-up. In: Welge-Luessen A, Hummel, T, eds. Management of Smell and Taste Disorders: A Practical Guide for Clinicians. Stuttgart, Germany: Thieme; 2013:49-57.

42. Hummel T, Hummel C, Welge-Luessen A. Assessment of olfaction and gustation. In: Welge-Luessen A, Hummel T, eds. Management of Smell and Taste Disorders: A Practical Guide for Clinicians. Stuttgart, Germany: Thieme; 2013:58-75.

43. Landis BN, Welge-Luessen A, Bramerson A, et al. "Taste Strips"—a rapid lateralized, gustatory bedside identification test based on impregnated filter papers. J Neurol 2009;256:242-248.

44. Hummel T, Sekinger B, Wolf SR, Pauli E, Kobal G. 'Sniffin' sticks': olfactory performance assessed by the combined testing of odor identification, odor discrimination and olfactory threshold. Chem Senses 1997;22:39-52.

45. Smutzer G, Desai H, Coldwell SE, Griffith JW. Validation of edible taste strips for assessing PROP taste perception. Chem Senses 2013;38: 529-539.

46. Hayes JE, Bartoshuk LM, Kidd JR, Duffy VB. Supertasting and PROP bitterness depends on more than the TAS2R38 gene. Chem Senses 2008;33: $255-265$.

47. Sandell M, Hoppu U, Mikkilä V, et al. Genetic variation in the hTAS2R38 taste receptor and food consumption among Finnish adults. Genes Nutr 2014;9:433.

48. Rousset F. GENEPOP'007: a complete re-implementation of the GENEPOP software for Windows and Linux. Mol Ecol Resour 2008;8:103-106.

49. Engels WR. Exact tests for Hardy-Weinberg proportions. Genetics 2009;183: 1431-1441.

50. Guo SW, Reed DR. The genetics of phenylthiocarbamide perception. Ann Hum Biol 2001;28:111-142. 